\title{
Increased trace amine-associated receptor 1 (TAAR1) expression is associated with a positive survival rate in patients with breast cancer
}

\author{
Aurelia Vattai ${ }^{1}$ *Elif Akyol ${ }^{1}$ Christina Kuhn ${ }^{1}$ *Simone Hofmann ${ }^{1}$ Helene Heidegger ${ }^{1}{ }^{*}$ Franz von Koch ${ }^{4}$ - \\ Kerstin Hermelink $^{1} *$ Rachel Wuerstlein ${ }^{1}$ *Nadia Harbeck ${ }^{1} *$ Doris Mayr $^{3} *$ Christine Spitzweg $^{2} \cdot$ Bettina Toth $^{3}$ * \\ Sven Mahner ${ }^{1} \cdot$ Udo Jeschke $^{1} \cdot$ Nina Ditsch $^{1}$
}

\begin{abstract}
Purpose A correlation between breast cancer and thyroid disorders has been described in previous studies. Degraded thyroid hormones are referred to as trace amines. These endogenous amines have the ability to bind to the G-protein-coupled receptor TAAR1 (trace amine-associated receptor) and thereby activate it. TAAR1 is able to modulate the serotonergic and dopaminergic system in the brain and has so far been studied in the neurological field. The following study represents the first investigation of the regulation of TAAR1 in primary breast cancer (no metastases, MO).

Methods Immunohistochemical analyses were carried out to detect TAAR1 expression in formalin fixed paraffin embedded breast cancer samples. Survival times of primary breast cancer patients (MO) with and without TAAR1 expression in their tumours were compared by Kaplan-Meier curves, and correlations between ordinal
\end{abstract}

Udo Jeschke

Udo.Jeschke@med.uni-muenchen.de

1 Department of Gynaecology and Obstetrics, Breast Center, Ludwig-Maximilians University of Munich, Marchioninistrasse 15, 81377 Munich, Germany

2 Department of Internal Medicine, Ludwig-Maximilians University of Munich, Marchioninistrasse 15, 81377 Munich, Germany

3 Department of Gynaecological Endocrinology and Reproductive Medicine, University Hospital Innsbruck, AnichstraßBe 35, 6020 Innsbruck, Austria

4 Department of Obstetrics and Gynaecology, Klinikum Dritter Orden, Menzinger Str. 44, 80638 Munich, Germany

5 Department of Pathology, Ludwig-Maximilians University of Munich, Thalkirchner Str. 142, 81337 Munich, Germany variables were determined with Spearman's rank correlation coefficient.

Results The investigation showed a correlation between TAAR1 expression and tumour differentiation grade. A well differentiated tumour grade (G1) was associated with higher TAAR1 expression and HER2 and HER4 positivity predicted higher TAAR1 expression. A TAAR1 overexpression (IRS $\geq 6$ ) was associated with significantly longer overall survival (OS) $(p=0.02)$ than that of reduced TAAR1 expression (IRS $<6$ ) during a maximum followup of 14 years, demonstrating that TAAR1 has a favourable effect on OS of early breast cancer patients.

Conclusions We conclude that TAAR1 seems to be an independent predictor for breast cancer survival. Modulation of TAAR1 may represent a novel targeting strategy for breast cancer prevention and therapy.

Keywords TAAR1 (trace amine-associated receptor 1 ) Breast cancer $\bullet$ Trace amines $\bullet$ L-DDopa decarboxylase • HER2 • HER4

\section{Purpose}

Breast cancer is the most common malignant tumour in female patients worldwide and one of the three most common cancers worldwide along with colon- and lung cancer (Harbeck and Gnant 2017). In 2012, about 1.7 million patients worldwide were diagnosed with breast cancer, and almost half a million people died from it (Torre et al. 2015; Ferlay et al. 2015). There is evidence that breast cancer and thyroid disorders correlate and patients with thyroid dysfunctions show higher incidences of breast cancer in comparison to healthy controls (Türken et al. 2003; Rasmusson et al. 1987; Kuijpens 
et al. 2005). However, the detailed role of thyroidal hormones in breast cancer is still unclear, and future investigations are necessary (Heublein et al. 2015). In a recently published study, an increased risk of breast cancer in women with hyperthyroidism and a decreased risk in women with hypothyroidism could be shown, thereby indicating an association between thyroidal function and breast cancer risk (Sogaard et al. 2016). Furthermore, fT4 and TSH levels seem to be predictive for therapeutic response and prognosis of patients with recurrent breast cancer (Yokoe et al. 1996). In a prospective study, Ditsch et al. (2010) showed that blood levels of $\mathrm{fT}_{3}$ and $\mathrm{fT}_{4}$, and concentrations of antibodies against thyroidal peroxidase and TSH are significantly elevated at the time point of primary diagnosis of breast cancer.

Thyroid hormones bind to the thyroid hormone receptor (THR), which is a member of the nuclear receptor superfamily. Nuclear receptors play a role in numerous physiological processes, and they act via transcriptional cis-regulation of target genes (Pestka et al. 2013). Thyroid hormones regulate numerous genes that are involved in cell differentiation, proliferation and apoptosis (Perri et al. 2014; Chi et al. 2013). Ditsch et al. (2013) analysed THR expression in breast cancer patients. The isoform THRa2 was found to be significantly associated with prognostic histopathological parameters such as tumour size, axillary lymph node involvement, tumour grade and hormone receptor status (Ditsch et al. 2013). Via a multivariate analysis, a trend for THRa 2 as an independent predictor of disease-free and overall survival could be shown (Ditsch et al. 2013). Hence, patients with a higher expression of THRa 2 in their tumours have a better prognosis than patients with low THRa 2 levels (Ditsch et al. 2013). In addition, THR $\beta$ is a positive prognostic factor for overall survival at 5 years and overall survival (OS), while THRa positivity predicts a reduced overall survival at 5 years (Heublein et al. 2015). Heublein et al. (2015) assume that THR $\alpha$ and THR $\beta$ represent interesting alternative targets for endocrine treatment of $B R C A I$ associated triple negative breast cancer.

The degradation of thyroid hormones results in the formation of trace amines (TAs), which are endogenous amines (Harmeier et al. 2015). The enzyme L-dlopa-decarboxylase, also known as aromatic amino acid decarboxylase, is involved in the transformation of thyroxine (T4) into 3-iodothyronamine (TjAM) (Sumi-Ichinose et al. 1992; Le Van Thai et al. 1993). During the transformation of thyroxine into 3-iodothyronamine, a deiodisation and a decarboxylation take place (Sumi-Ichinose et al. 1992; Le Van Thai et al. 1993). The enzyme L-dloprat-decarboxylase is also involved in the synthesis of dopamine, serotonin, tryptamine, phenylethylamine and histamine (The Human Metabolome Database 2016). A deficiency in L-dlopa-decarboxylase (AADC) is associated with severe developmental delay, oculogyric crises and autonomic dysfunction (Pons et al. 2004).

Classical thyroid hormones act rather epigenetically, while decarboxylated thyroid hormones act in the opposite direction through rapid actions, such as rapid lowering of body temperature and heart rate (Brix et al. 2011). Future investigations are necessary to further clarify the physiological role of 3-iodothyronamine-TANA1 signalling (Wang et al. 2014). Up to now, no data exist in the current literature about the influence of thyroid hormone dérivâtes and their receptors on breast cancer outcome.

Thyronamines, the thyroid hormone dérivâtes, function via the activation of a G-protein coupled receptor, called trace amine-associated receptor 1 (TAAR1) (Brix et al. 2011). TAAR1 was first described in 2001 by Borowsky et al. (2001). Upon activation of adenylcyclase, the receptor induces a rise of intracellular cAMP level (Borowsky et al. 2001; Barak et al. 2008). TAAR1 is expressed by numerous organs such as the placenta, brain, spinal cord, stomach, pancreatic $\boldsymbol{B}$-cells and in immune cells like macrophages, leukocytes and dendritic cells (Lam et al. 2015; Gozal et al. 2014; Babusyte et al. 2013). TAAR1 can be activated by different compounds, including dopaminergic, adrenergic and serotonergic classes (Sotnikova et al. 2009). A wide spectrum of agonists, such as trace amines, common biogenic amines, amphetamine-like stimulants and thyroid hormone dérivâtes like 3-iodothyronamine have the ability to activate TAAR1 (Scanlan et al. 2004; Bunzow et al. 2001).

So far, TAMR1 has been investigated in the neurological field as TAAR1 is able to modulate the serotonergic and dopaminergic system in the brain (Lam et al. 2015). As $T \mathbf{A A R} 1$ is a negative regulator of dopamine transmission, it represents a target for neuropsychiatrie disorders such as depression and Parkinson's disease (Lam et al. 2015). Furthermore, stimulation of TAMR1 has a beneficial effect on glucose- and obesity control in mice with type- 2 diabetes mellitus (Raab et al. 2016).

In addition to thyroid hormones, ErbB receptors play an important role in the pathogenesis of breast cancer (Karamouzis et al. 2007). The proto-oncogene ErbB receptors are members of the epidermal growth factor receptor (EGFR) family of receptor tyrosine kinases (Naresh et al. 2006), including ERBB2/HER2/neu (ERBB2), ERBB3 and ERBB4. They can influence cell proliferation, survival and differentiation (Karamouzis et al. 2007; Zoi et al. 2016). ERBB2 is responsible for the progression of primary tumours, and its expression is associated with poor prognosis of breast cancer patients (Yarden and Sliwkowski 2001; Tan and Yu 2007). In recent years, especially ERBB2 has become an important biomarker and target in the treatment of breast cancer (Mitri et al. 2012; Witzel and Muller 2015). Treatment with the monoclonal antibody 
Trastuzumab (Herceptin) is recommended in patients with HER2-positive early breast cancer for 1 year (Mates et al. 2015). In June 2012, the FDA approved the monoclonal

Table 1 Patients' characteristics

\begin{tabular}{|c|c|c|}
\hline & $n$ & $\%$ \\
\hline \multicolumn{3}{|l|}{ Histology } \\
\hline NST & 131 & 57.7 \\
\hline Non & 96 & 42.3 \\
\hline \multicolumn{3}{|l|}{ Tumour grade } \\
\hline $\mathrm{G} 1, \mathbf{G} 2$ & 103 & 45.4 \\
\hline G3 & 53 & 23.3 \\
\hline NA (not available) & 71 & 31.3 \\
\hline \multicolumn{3}{|l|}{$\mathrm{pT}$} \\
\hline PT1 & 154 & 67.8 \\
\hline pT2-pT4 & 72 & 31.7 \\
\hline NA & 1 & 0.5 \\
\hline \multicolumn{3}{|l|}{ pN } \\
\hline pNO & 122 & 53.7 \\
\hline pN1 & 93 & 41.0 \\
\hline NA & 12 & 5.30 \\
\hline \multicolumn{3}{|l|}{ CIS } \\
\hline No & 107 & 47.1 \\
\hline Yes & 120 & 52.9 \\
\hline \multicolumn{3}{|l|}{ ER } \\
\hline Negative & 30 & 13.2 \\
\hline Positive & 175 & 77.1 \\
\hline NA & 22 & 9.7 \\
\hline \multicolumn{3}{|l|}{ PR } \\
\hline Negative & 62 & 27.3 \\
\hline Positive & 128 & 56.4 \\
\hline NA & 37 & 16.3 \\
\hline \multicolumn{3}{|l|}{ HER2 } \\
\hline Negative & 160 & 70.5 \\
\hline Positive & 20 & 8.8 \\
\hline NA & 47 & 20.7 \\
\hline \multicolumn{3}{|l|}{ HER4 } \\
\hline Negative & 201 & 88.6 \\
\hline Positive & 16 & 7.0 \\
\hline NA & 10 & 4.4 \\
\hline \multicolumn{3}{|l|}{ TAAR1 } \\
\hline Negative & 95 & 41.9 \\
\hline Positive & 127 & 55.9 \\
\hline NA & 5 & 2.2 \\
\hline \multicolumn{3}{|l|}{ Age } \\
\hline$\leq 55$ years & 90 & 39.6 \\
\hline$>55$ years & 136 & 60.0 \\
\hline nNA & 1 & 0.4 \\
\hline
\end{tabular}

In total, 227 patients were included in the study. AH patients are MO (no metastases) antibody Pertuzumab, which inhibits the dimerization between HER2 and HER3 receptors, as a combination therapy together with Trastuzumab in metastatic breast cancer (USFaD Administration 2016).

The aim of our study was the analysis of TAAR 1 expression in breast cancer tissue and the correlation of TAAR1 expression with survival of breast cancer patients. An additional aim was the assessment of the association of TAAR1 expression with the expression of ErbB receptors. As TAAR1 has not been described in breast cancer tissue before, this investigation represents the first analysis of TAARI regulation in breast cancer.

\section{Methods}

\section{Patients' and specimens' characteristics}

Formalin fixed paraffin embedded (FFPE) primary breast cancer samples of 227 patients (all MO) who had undergone surgery for a malignant breast tumour at the Department of Gynaecology and Obstetrics, Ludwig-Maximilians-Umiversity Munich, Germany, from 1998 until 2000 were included in the study. Women with benign tumours of the breast were excluded from the study. Histopathological tumour subtypes were assigned according to the WHO criteria, and tumour grade $(\mathbf{G 1}, 2,3)$ was determined according to the Elston and Ellis criteria (1993) by a gynaecological pathologist (Table 1). Tumour grade is a measure of the cell appearance and increases with the lack of cellular differentiation. The grading system includes G1: well differentiated, G2: moderately differentiated and G3: poorly differentiated. Clinical and follow-up data regarding patient age, overall survival and relapse free survival, lymph node status, presence of metastases, ER/PR results and HER2 and HER4 detection were retrieved from patients' charts and from the Munich Cancer Registry. HER 2 positivity is clearly defined by the DAKO Scoring system (DAKO, HER2 FISH pharmDx ${ }^{\mathrm{TM}}$ Kit). As HER2 status was not determined routinely in Germany before 2001, it was retrospectively assessed for patients who had surgery before 2001. HER2 status was determined as recommended in the national guidelines, i.e., by DAKO Score and FISH analysis in cases of DAKO $2+$.

\section{Immunohistochemistry}

Expression of the G-protein coupled receptor TAAR1 and the ErbB receptors HER2 and HER4 was identified immunohistochemically from the embedded breast cancer samples. Tissue samples were fixed in neutral-buffered formalin $(3.7 \%)$ right after resection and they underwent standardised paraffin embedding. For immunohistochemistryy, FFPE tissue sections $(3 \mathrm{pm})$ were deparaffinised in xylol, rehydrated 
in a descending ethanol gradient and prepared for epitope retrieval in a pressure cooker using sodium citrate buffer (pH 6.0). Next, sections were blocked with $3 \% \mathrm{H}_{2} \mathrm{O}_{2}$ in methanol $(20 \mathrm{~min})$ at room temperature for the inactivation of the endogenous peroxidase. Blocking solution was applied for blocking of the non-specific binding of the primary antibodies. Sections were then incubated with the following primary antibodies consecutively: Anti-TAAR1 (polyclonal rabbit IgG, Abeam, Cambridge, UK), anti-HER2 (c-erbB-2 oncoprotein, polyclonal rabbit IgG, Dako, Santa Clara, USA), anti-HER4 (polyclonal rabbit IgG, Epitomics, Burlingame, USA) and phosphorylated anti-HER4 (polyclonal rabbit IgG, Epitomics, Burlingame, USA). Antibody reactivity was detected using the Vectastain Elite ABC-Kit (Vector Laboratories, Burlingame, USA) according to the manufacturer's protocol. In the following step, substrate and chromogen (3,3'-diaminobenzidine DAB; Dako, Glostrup, Denmark) were added to the slides, which were then counterstained with Mayer's acidic haematoxylin and cover slipped. Appropriate positive (samples from the sigma) and negative controls were included in each experiment (Fig. 1).

Breast cancer sections were examined by two independent observers using a Leitz Diaplan microscope (Leitz, Wetzlar, Germany). For each slide, tumour tissue was examined independently by applying the semiquantitative immunoreactive score (IRS) which optically estimates the intensity and distribution pattern of antigen expression (Remmele and Stegner 1987). The IRS is calculated by multiplying the percentage of positively stained cells ( 0 : no staining; 1: $\leq 10 \%$ of the cells; 2: 11-50\%; 3: 51-80\%, 4: $>80 \%$ ) with the intensity of the cells' staining ( 0 : none; 1 : weak; 2: moderate; 3 : strong). A receiver operating characteristic curve (ROC curve) was used to determine the cut-off level between TAAR1 overexpression and reduced TAAR1 expression. For identification of the cut-off level, the maximum difference between sensitivity and specificity was used. Images were taken with a CCD colour camera (JVC, Victor Company of Japan, Japan).

\section{Statistical analysis}

SPSS statistics 22 (Armonk, NY: IBM Corp.) was used for data analysis, $\mathbb{p}$ values lower than 0.05 were considered to be statistically significant. Survival times were compared by Kaplan-Meier analysis, and differences in the patients" overall survival times were tested for significance by Cox Mantel log-rank test. Group comparisons regarding ordinal analysis variables were tested with Mann-Whitney $\boldsymbol{U}$ tests or Kruskal-allis tests as appropriate. Correlations between ordinal variables were tested with Spearman's rank correlation coefficient. Cox-regression analysis was used to compare the risk of death in patients with and without TAAR1 expression when the effects of further factors were accounted for. Independent variables included in the Cox-regression model were TAAR1 expression (IRS $>6$ ), histological subtype, tumour grade, tumour size, lymph node status, hormone receptor status (ER/PR), HER2
Fig. 1 Negative and positive controls of TAAR1 (Sigma), a Negative isotype control of TAAR1, sigma 1872-02; x110 magnification; b negative isotype control of TAAR 1 , sigma 1872-02; x25 magnification; c positive isotype control of TAAR1, sigma 1872-02; x110 magnification; $\mathbf{d}$ positive isotype control of TAAR1, sigma 1872-02; x25 magnification
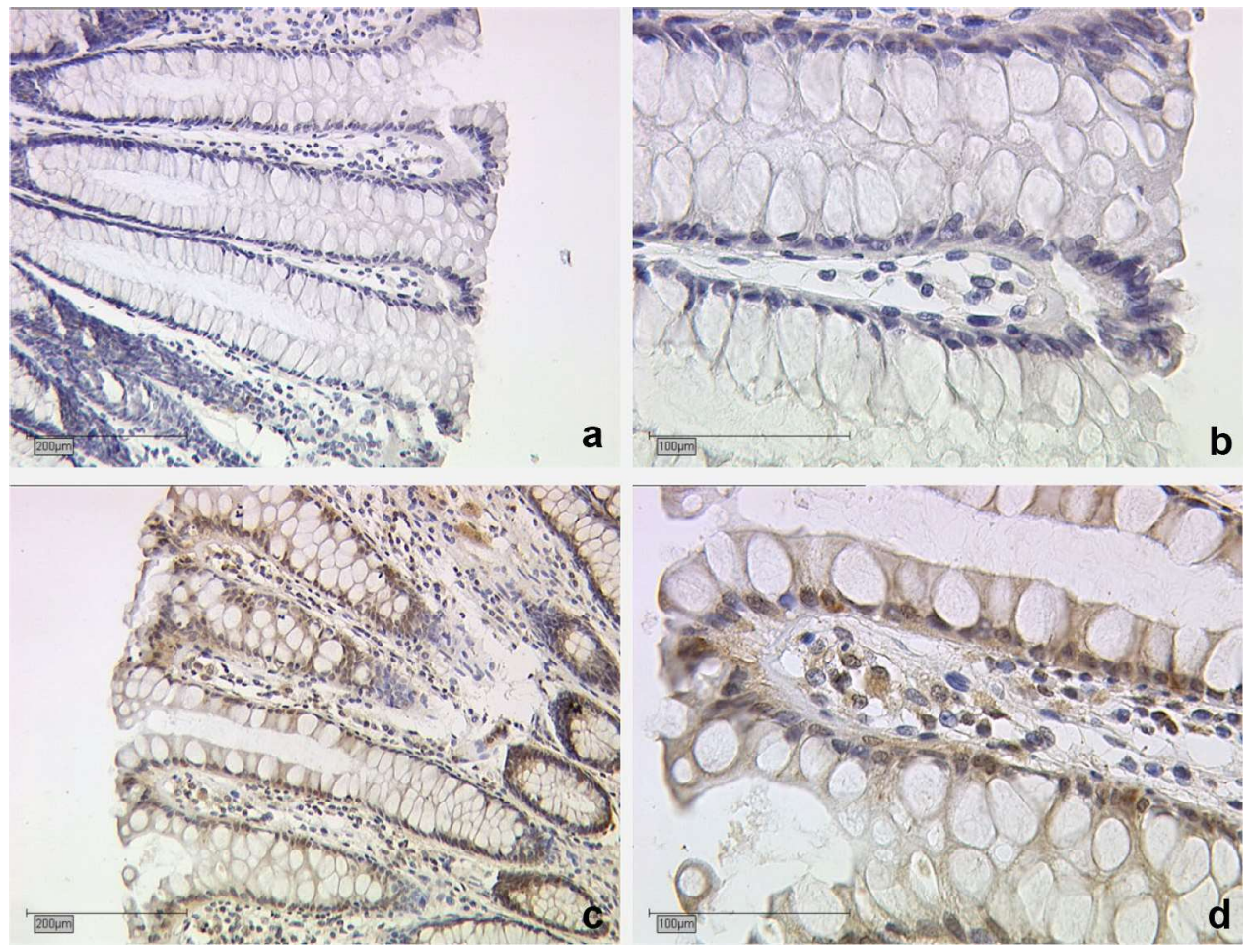
status, HER4 and phosphorylated HER4 status and age at surgery.

\section{Results}

Clinical and histopathological data of the patient cohort

In total, 227 patients were included in the study. The clinical and histopathological data of the patient cohort are shown in Table 1.90 patients $(39.6 \%)$ were 55 years or younger, and 136 patients $(60.0 \%)$ were older than 55 . Most of the patients had a pT1 tumour size $(n=154$; $67.8 \%$ ) and the rest of the patients had a pT2-pT4 tumour $(n=72,31.7 \%)$. Tumour size data of one patient was not available $(n=1,(0.5 \%)$. G1 or G2 tumour grade were found in 103 tumours $(45.4 \%)$ and 53 tumours $(23.3 \%)$ had a G3 differentiation grade. The tumour grade of lobular cancer was formerly not assessed; therefore, 71 tumours (31.3\%) were not classified by tumour grade. 122 patients $(53.7 \%)$ were lymph node negative in comparison to 93 patients (41.0\%) with positive lymph nodes. The lymph node status of five patients $(5.3 \%)$ was missing. With regard to ER/PR status, most patients had hormone receptor positive tumours (ER 77.1\%, PR 56.4\%). In total, 227 samples have been analysed according to the HER2-status. 23 samples were HER2-positive (10.1\%), 181 samples (79.8\%) were HER2-negative and 23 samples (10.1\%) could not be assessed properly. The HER2-status was not assessed as part of the clinical routine; therefore, we analysed HER2 expression retrospectively in cooperation with the Institute of Pathology. 201 tumours were HER4-negative (88.6\%), 16 tumours were HER4-positive (7.0\%) and 10 tumours were not assessed $(4.4 \%)$.

\section{Expression of TAAR1 in different breast cancer subtypes and classification of TAAR1 expression by tumour grade}

TAAR1 was detected in 127 cases (55.9\%), and 95 cases (41.9\%) showed no TAAR1 expression in their primary breast tumour. Five cases $(2.2 \%)$ could not be assessed. In Table 2 the distribution of TAAR1 IRS score is shown. Figure 2 shows a boxplot analysis of TAAR1 expression (quantified by IRS) in different breast cancer subtypes of the collective. TAAR1 expression was most frequently found in breast cancer not otherwise specified (NST; $n=126,55.6 \%$ ), followed by invasive lobular cancer ( $n=47,20.7 \%)$, and medullary breast cancer $(n=20$, 8.8\%). This difference, however, was not significant. A median IRS of 4 of the TAAR1 expression was observed in invasive lobular and tubular cancer. TAAR1 expression
Talblk2 Distribution of TAAR1 IRS-Scores

\begin{tabular}{lrr}
\hline IRS & \multicolumn{1}{c}{$\boldsymbol{n}$} & $\%$ \\
\hline 0 & 9 & 4.0 \\
1 & 9 & 4.0 \\
2 & 27 & 11.9 \\
3 & 23 & 10.1 \\
4 & 27 & 11.9 \\
6 & 28 & 12.3 \\
8 & 64 & 28.2 \\
9 & 12 & 5.3 \\
12 & 23 & 10.1 \\
NA & 5 & 2.2 \\
\hline
\end{tabular}

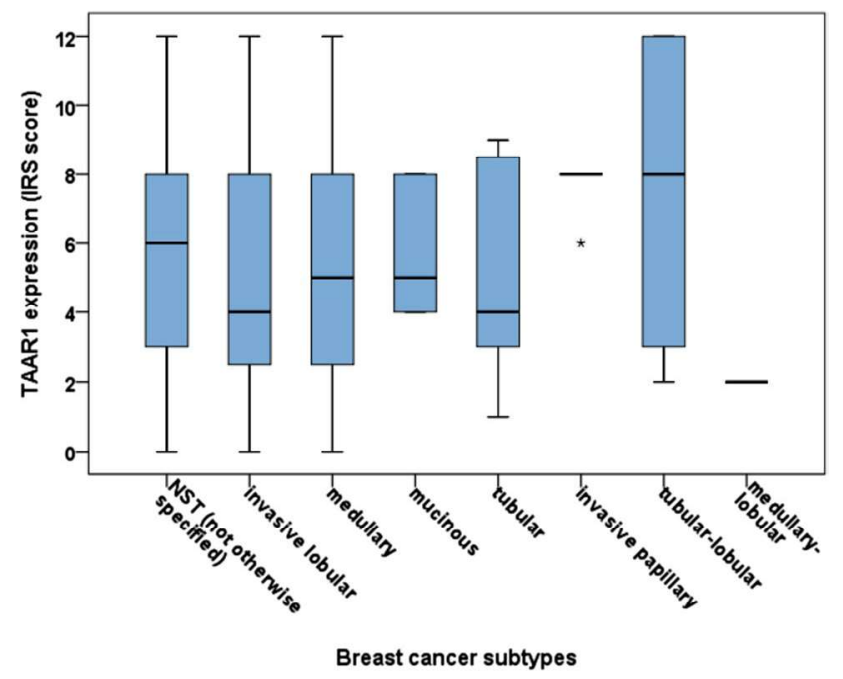

Fig. 2 Expression of TAAR1 in different breast cancer subtypes. NST, $n=126$; invasive-lobular, $n=47$; medullary, $n=20$; mucinous, $n=6$; tubular, $n=7$; invasive-papillary, $n=5$; tubular-lobular, $n=10$; medullary-lobular, $n=1$. Median TAAR1 expression is highest in NST (IRS 6) and the tubular-lobular subtype (IRS 8)

with a higher median IRS of 8 was observed in the invasive-papillary and tubular-lobular breast cancer.

Furthermore, the association of TAAR1 expression and the tumour grade was tested. Seventeen (7.5\%), 86 (37.9\%) and $53(23.3 \%)$ cases had a tumour grading of 1,2 and 3 , respectively (Fig. 3). TAMR1 expression correlated with tumour grade. Breast cancer samples with a tumour grade of 1 showed a median IRS TAAR1 staining of 8, whereas a median IRS TAAR1 staining of 6 was found in grade 2 as well as in grade 3 tumours.

\section{Positive correlation of TANR1 with HER2 and HER4 expression in breast cancer patients}

For both HER receptors, a correlation with TAAR1 expression was identified. HER2-positive breast cancers 
Fig. 3 a Expression of TAAR1 in different breast cancer types classified by tumour grade $(1=\mathrm{G} 1,2=\mathrm{G} 2,3=\mathrm{G} 3) ; \mathbf{b}$ TAAR1 nuclear expression in breast cancer, low expression, magnification $x$ 10; c TAAR1 nuclear expression in breast cancer, low expression, magnification $\times 25$; $d$ TAAR1 cytoplasmic expression in breast cancer, high expression, magnification x 10; e TAAR1 cytoplasmic expression in breast cancer, high expression, magnification $\times 25$
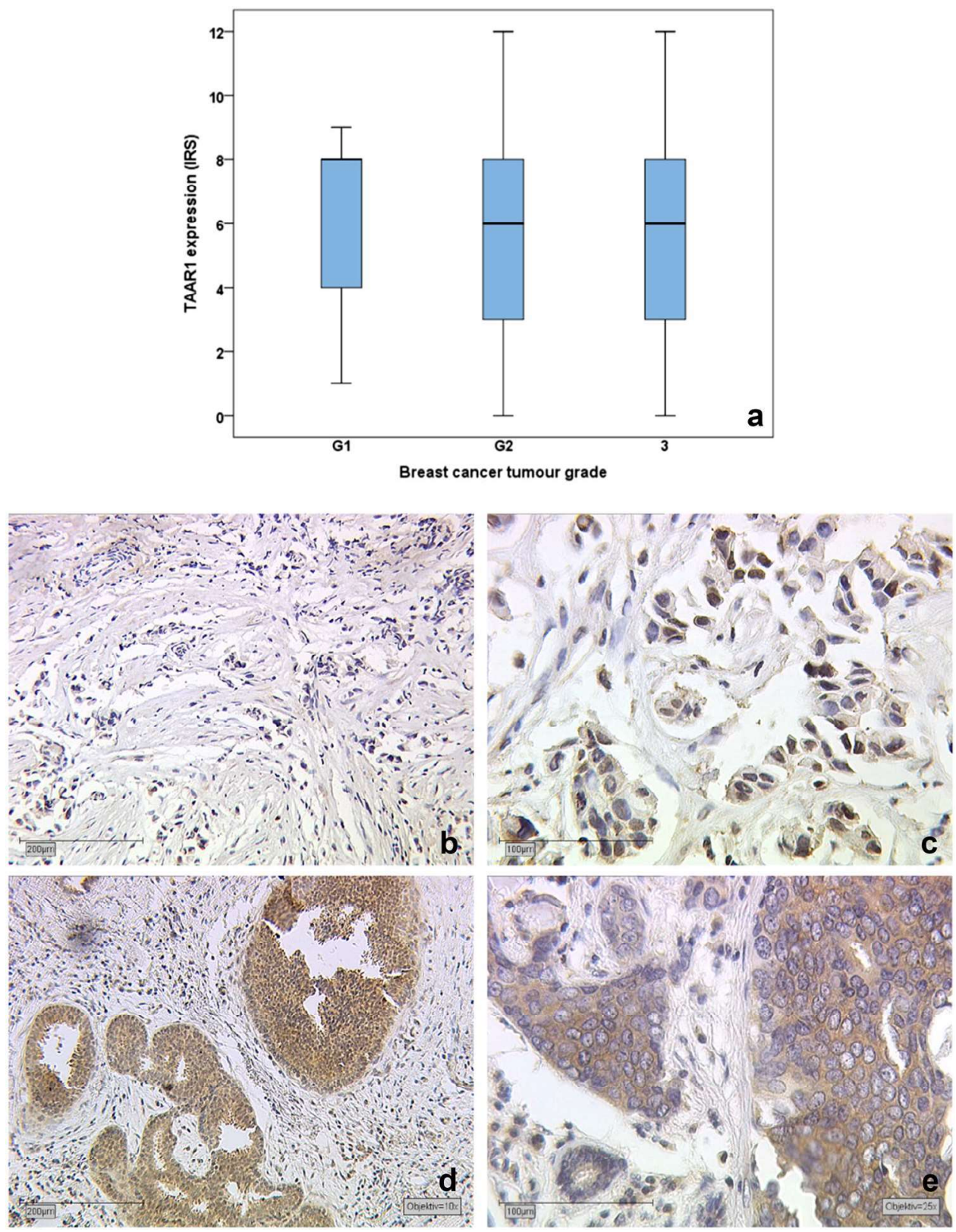

showed a significantly higher TAAR1 expression (median IRS $8 ; n=23$ ) in comparison to HER2-negative tumours (median IRS $6 ; n=181 ; p<0.001$ ) (Fig. 4). Hence, HER2 positivity is associated with an increased expression of TAARI.

In breast cancer patients with HER4-positive breast tumours, the same trend could be shown as demonstrated for HER2-positive tumours (Fig. 5). The median IRS TAAR1 was 4 in HER4-negative tumours $(n=108)$ and 8 in HER4-positive tumours $(n=114)$ with a $p$ value $<0.001$.
Association of TAAR1 expression (IRS $\geq 6$ ) with a more favourable overall survival rate of breast cancer patients

Patients with a positive staining of TAARI in breast cancer tissue were compared to patients with a negative TAAR1 staining regarding their overall survival rate. Patients with tumours showing a TAAR1 IRS greater than or equal to 6 had a more favourable OS in comparison to patients with lower TAAR1 expression $(p=0.04)$. The significant difference is shown in the Kaplan-Meier 

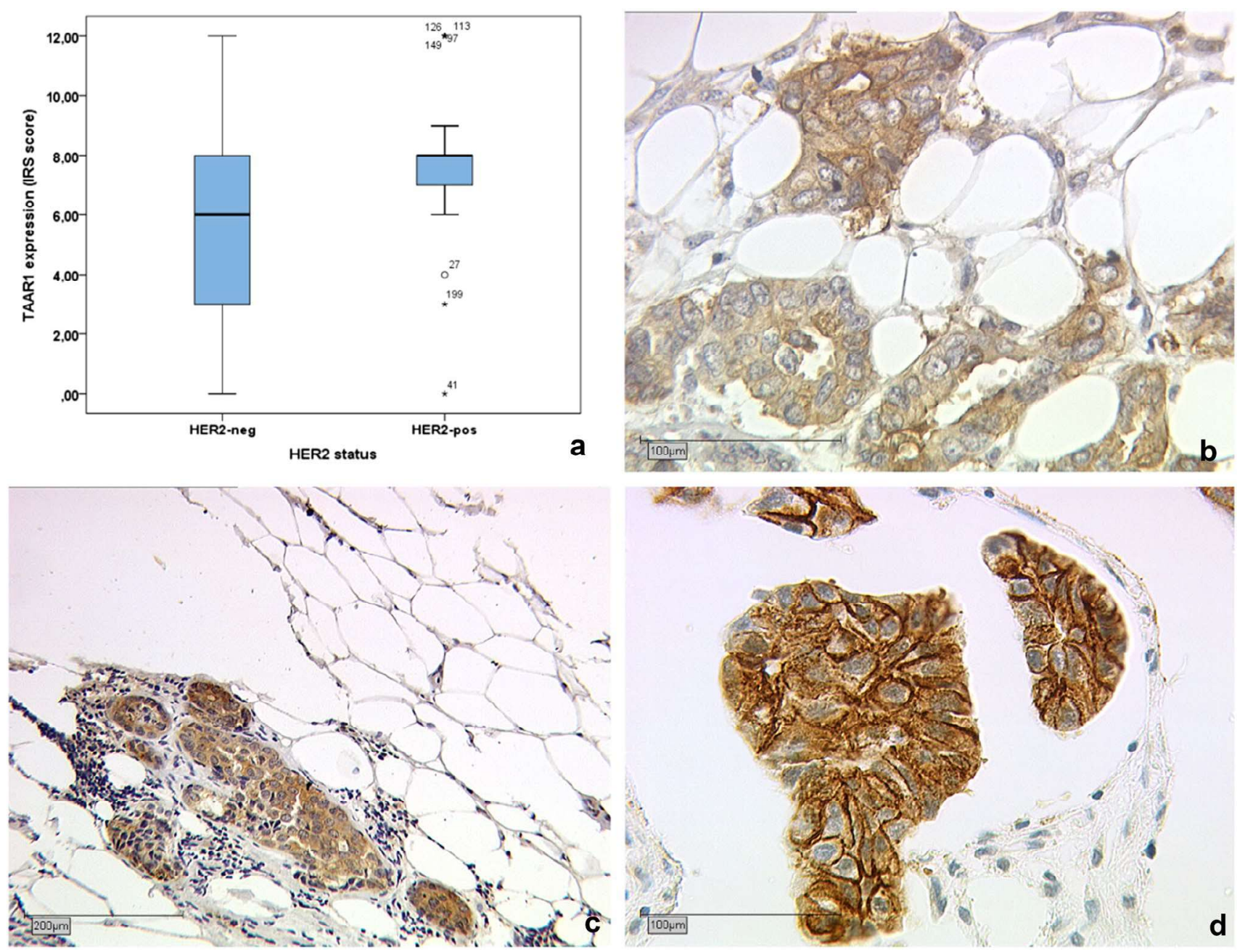

Fig. 4 Correlation of TAAR1 with HER2. a Box $p l o t$ analysis of TAAR1 and HER2 correlation $(p<0.001)$; HER2-negative, $n=181$; HER2positive, $n=23$. b TAAR1 expression, magnification $\times 10$; $\mathbf{c}$ HER2 expression, magnification $\times 10$; $d$ HER2 expression, magnification $\times 25$

curve in Fig. 6. TAAR1 therefore seems to be a positive prognosticator for overall survival of breast cancer patients.

TAAR1 expression with an IRS $* 6$ showed a median overall survival of 8 years with a box length between 4 and 13 years and TAAR1 IRS score $\geq \mathbf{6}$ showed a median overall survival of 9 years with a box length between 7 and 13 years (Fig. 7).

According to the nodal status, a negative nodal status (pNO) showed a median overall survival of 10 years with a box length between 8 and 12 years and a positive nodal status (pN1) showed a median overall survival status showed a median overall survival of 8 years with a box length between 3 and $\mathbf{1 2}$ years (Fig. 8).

\section{Cox-regression of histopathological variables and age on overall survival in breast cancer patients}

Multivariate Cox-regression was performed to test which histopathological variables are independent prognosticators for the overall survival (OS) rate in the tested breast cancer collective. It could be shown that TAAR1 expression $(p=0.021)$, lymph node status $(\mathrm{pN})(p<0.001)$ and age at surgery $(p=0.01)$ are independent prognosticators for the overall survival (Table 3 ). Breast cancer tissue has also been assessed for ER and PR status and TAAR1 expression. For both ER $(p=0.608)$ and PR $(p=0.597)$ no significant correlation with TAAR1 expression could be detected. No effect could furthermore be demonstrated 

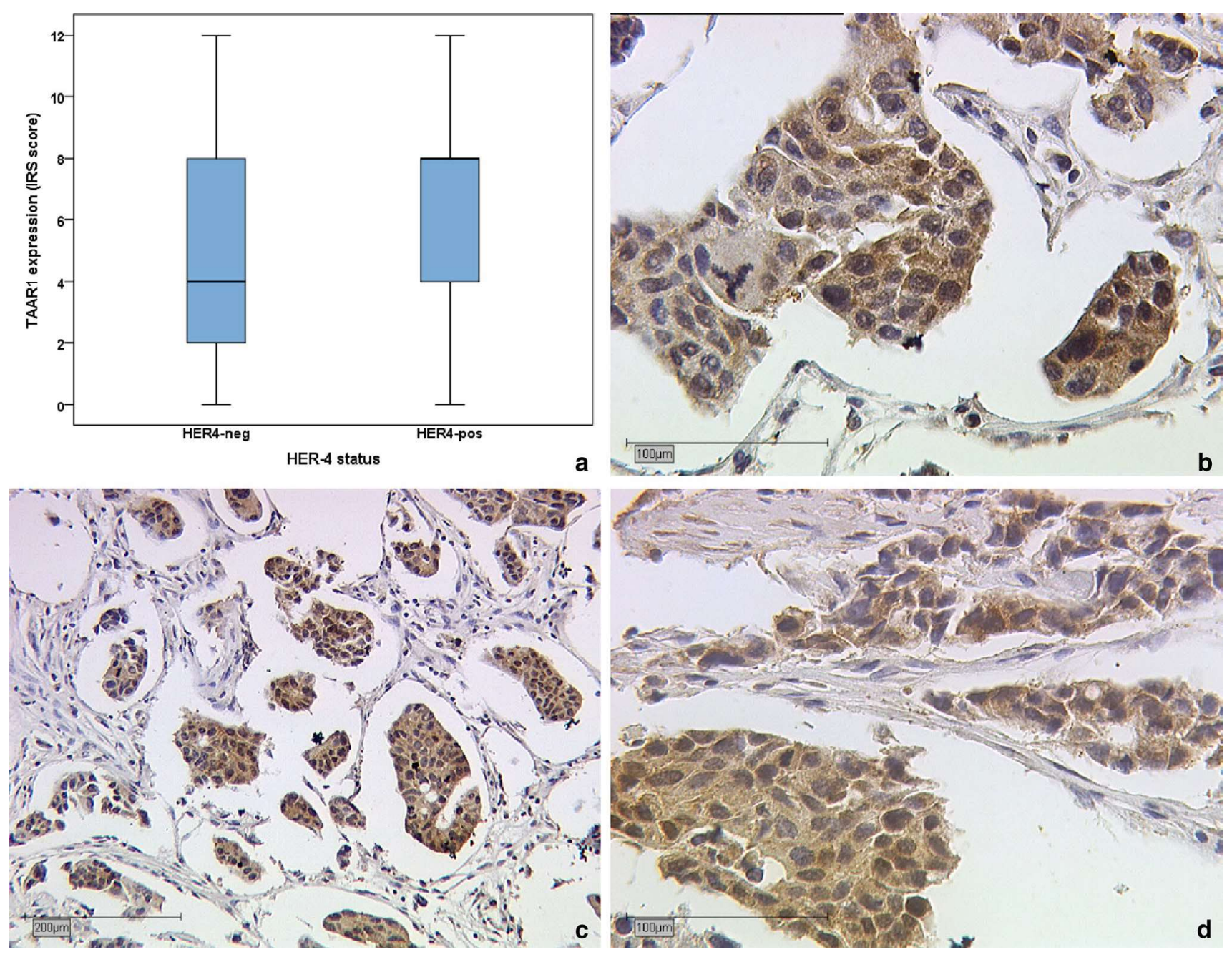

Fig. 5 Correlation of TAAR1 with HER4. a Box plot analysis of TAAR1 and HER4 correlation HER4-negative, $n=108$; HER4-positive, $n=114$. b TAAR1 expression, magnification $\times 25 ; \mathrm{c}$ HER4 expression, magnification $\times 10 ; \mathbf{d}$ HER4 expression, magnification $\times 25$

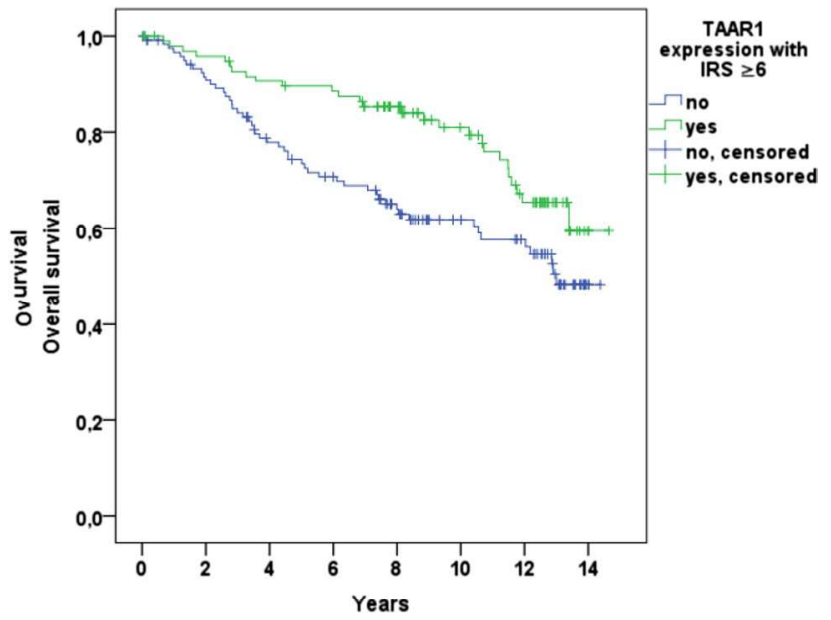

Fig. 6 Influence of TAAR1 expression on OS of patients with early breast cancer. Increased TAAR1 expression (IRS $\geq 6$ ) is associated with a longer overall survival $(p=0.04)$ for the other histopathological variables, such as histology, tumour grade, tumour size, ER and PR status, HER2-, HER4- and pHER4 status.

\section{Conclusion}

We examined TAAR1 expression in different histological subtypes of primary malignant breast cancer (MO). To our knowledge, this is the first time that associations of TAAR1 with other biological characteristics of breast cancer and the effect of TAAR1 overexpression on overall survival of breast cancer patients have been analysed.

A positive correlation between TAARI and HER2 status and HER4 status was present. Furthermore, a higher TAAR1 expression was associated with a positive HER2 status as well as staining of HER4 in primary breast cancers. Interactions between G-protein coupled receptors 


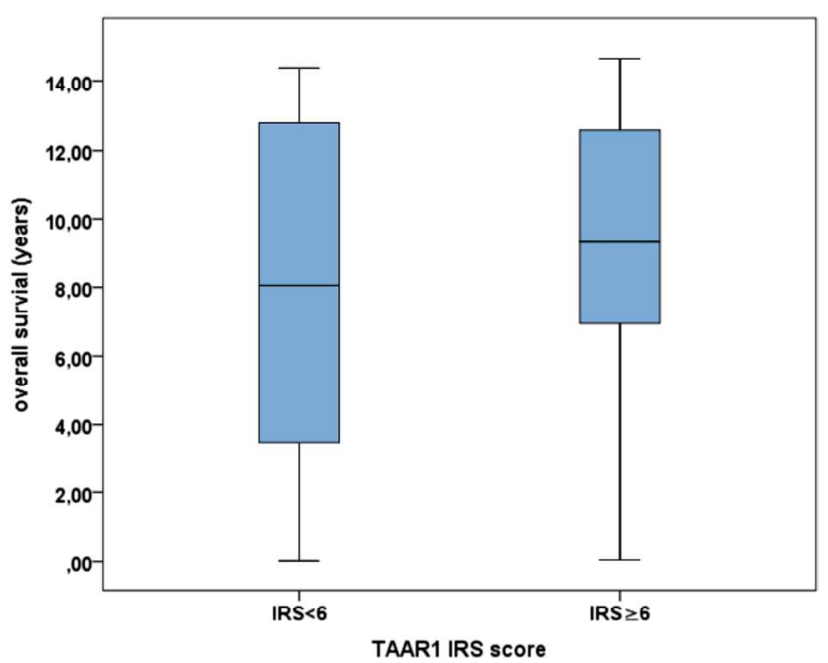

Fig-7 Correlation of TAAR1 IRS score and OS of patients with early breast cancer. The boxes represent the range between the 25th and 75 th percentiles with a horizontal line at the median. Hence, 50\% of the cases lie within the box. The bars delineate the 5th and 95th percentiles

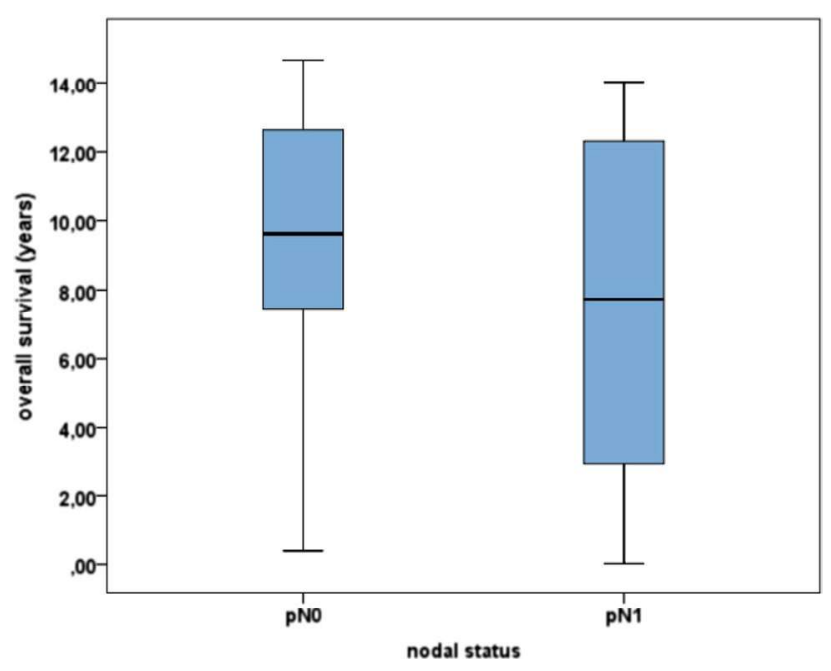

Fig. 8 Correlation of nodal status and OS of patients with early breast cancer. The boxes represent the range between the 25th and 75 th percentiles with a horizontal line at the median. Hence, $50 \%$ of the cases lie within the box. The bars delineate the 5th and 95th percentiles

and ErbB receptors have also been investigated by Pan et al. (2011), who assume that ErbB4 (HER4) signalling interacts with GPCRs. HER2 is known for its negative effect on the outcome of breast cancer, in particular before the advent of modem targeted therapies (Yarden and Sliwkowski 2001; Tan and Yu 2007). In contrast, HER4 has the ability to suppress tumour cell proliferation, and it has been suggested that HER4 may weaken HER2 signalling activity, which leads to the assumption that an overexpression of HER4 can inhibit breast cancer progression (Karamouzis et al. 2007; Barnes et al. 2005; Vidal et al. 2005). Naresh et al. (2006) suggest that HER4 suppresses breast cancer cell growth through the activation of the intrinsic apoptotic pathway. The positive correlation of TAAR 1 and HER4 expression, which has been detected in our study, is in line with the impact of HER4 on breast cancer cell growth which has been described in the literature and the influence of TAARI overexpression on overall survival demonstrated in this study.

An association between TAAR1 and tumour grade was found, showing that the levels of TAARI (quantified by IRS) inversely correlate with tumour grade. This is in line with the prognostic implications of higher TAMRI expression and lower tumour grade, which are both associated with better survival. A limitation to those findings is that tumour grade of lobular cancer was formerly not assessed and hence 71 patients of the cohort (31.3\%) were not classified by tumour grade and are, therefore, not included in the sub analysis.

We demonstrated for the first time that TAR1 overexpression (IRS $\geq 6$ ) has a favourable effect on overall survival of primary breast cancer patients. Increased $T \mathbb{A} \mathbb{A} 1$ expression correlates with a positive outcome of over-all survival of primary breast cancer patient. Upregulation of TAAR1 in breast cancer tissue can possibly induce an increased decarboxylisation of thyroid hormones, which may result in a positive effect on the outcome of breast cancer. One aim of future investigations should therefore be analysis of the enzyme L-dlopar-decarboxylase, which is responsible for the transformation of thyroid hormones into differentiated thyroid hormones (trace amines). Upregulation of TAMR1 is possibly based on an increased synthesis of biological active amines. Further studies are required to investigate the impact of TAMR1 ligands on the expression of TAMRI, and it is necessary to identify which ligand can lead to an upregulation of the expression of TAMR1 and of L-dlopa decarboxylase. Up to now, only little knowledge exists about the regulation of TAMR1 but latest research shows that TAAR1 can be upregulated through methamphetamine (Sriram et al. 2016). Sriram et al. (2016) could demonstrate an induction of TAAR1 mRNA expression in resting T-lymphocytes in response to methamphetamine. Treatment of the T-lymphocytes with methamphetamine for $6 \mathrm{~h}$ significantly increased TAMR1 mRNA expression $(p<0.001)$ and protein expression $(p<0.01)$ (Sriram et al. 2016). The research group suggests that TANR1 is likely to play an important role in methamphetamine-mediated immune modulatory responses (Sriram et al. 2016).

The present work demonstrates that TAAR1 is an independent prognosticator for the overall survival in breast cancer patients. A positive TAMR1 expression (vs. negative expression) is associated with better survival in 
Table 3 Cox-regression of histopathological variables in breast cancer regarding overall survival

\begin{tabular}{lllll}
\hline & Significance & Hazard ratio $(\operatorname{Exp}(\mathbf{B}))$ & Lower 95\% Cl of Exp(B) & Upper 95\% Cl of Exp(B) \\
\hline TAAR1 with IRS $>6$ & $\mathbf{0 . 0 2 1}$ & 0.35 & 0.573 & 1.530 \\
Histology (NST) & 0.243 & 1.06 & 0.938 & 1.055 \\
Tumour grade & 0.292 & 0.71 & 0.501 & 1.133 \\
PT & 0.622 & 1.18 & 0.771 & 1.955 \\
pN & $\mathbf{0 . 0 0 0}$ & 3.96 & 0.989 & 2.467 \\
ER & 0.872 & 1.00 & 0.988 & 1.002 \\
PR & 0.696 & 0.99 & 0.995 & 1.010 \\
HER2 & 0.343 & 1.26 & 0.652 & 3.560 \\
HER4 & 0.881 & 0.99 & 0.507 & 1.387 \\
pHER4 & 0.358 & 1.07 & 0.838 & 2.203 \\
Age at surgery & $\mathbf{0 . 0 1 2}$ & 1.05 & 0.997 & 1.040 \\
\hline
\end{tabular}

TAAR1 expression (IRS $>6$ ), lymph node status and age at surgery are independent prognostic markers for overall survival in breast cancer patients

The significant $p$-values of the independent prognostic markers for overall survival in breast cancer patients are marked in bold

breast cancer patients. Positive lymph node status and age at surgery represent additional independent histopathological parameters which prognosticate survival in our breast cancer cohort. Those results are in line with our earlier findings that showed that THR 2 is an independent prognostic factor for overall survival (Ditsch et al. 2013). Heublein et al. (2015) compared 5 year survival in THRa and THRR positive cases of sporadic breast cancer patients and BRCA1 carriers. That study demonstrated that THRa positivity was associated with a significantly reduced 5-year survival rate in BRCAI carriers, while THRa had no effect on patient survival in sporadic cancer cases. In contrast, THRR $\beta$ positivity was associated with a significantly higher 5-year survival rate in BRCAA-associated cancers, while THRB had no significant impact on survival of sporadic breast cancer (Heublein et al. 2015). Further studies are required to investigate protein and gene detection. Moreover, patient cohorts that are clinically well defined according to biological subtype and potentially confounding systemic therapy are needed in order to make definitive statements concerning TAAR1 as a prognostic factor in breast cancer.

In conclusion, TAAR1 is expressed by breast cancer tissue, and in our hypothesis-generating study, it could be identified as an independent predictor for breast cancer survival. Increased TAAR1 expression correlates with a favourable overall survival in patients with early breast cancer. In future, modulation of TAAR1 may represent an interesting and novel targeting strategy for breast cancer prevention and therapy. To permit valid conclusions, additional prospective studies with larger und clinically better defined patient cohorts are necessary to confirm the prognostic relevance of TAARI in breast cancer. Furthermore, studies are required to investigate the influence of TAAR1 ligands such as $\mathrm{T}_{\mathrm{i}} \mathrm{AM}$ on the expression of TAAR1 in breast cancer and to identify which ligand has the ability to increase TAAR1 expression in breast cancer.

Acknowledgements We thank Sandra Schulze for her excellent technical assistance and Laurent Soussana for proof reading the research article. This work was supported by the Bayerische Gleichstellungsförderung scholarship of Bavaria. The funder had no role in study design, data collection and analysis, decision to publish or preparation of the manuscript.

\section{Compliance with ethical standards}

Funding The study was been funded by the Bayerische Gleichstellungsförderung 2016 of Bavaria.

Conflict of interest We, the authors, declare that we have no conflict of interest.

Ethical approval All procedures performed in studies involving human participants were in accordance with the ethical standards of the institutional and/or national research committee and with the 1964 Helsinki declaration and its later amendments or comparable ethical standards.

Informed consent Informed consent was obtained from all individual participants included in the study. Prior to surgery, the patients gave their informed consent for their tissue to be used for research and future scientific work.

\section{References}

Babusyte A et al (2013) Biogenic amines activate blood leukocytes via trace amine-associated receptors TAAR1 and TAAR2. J Leukoc Biol 93(3):387-394 
Barak LS et al (2008) Pharmacological characterization of membraneexpressed human trace amine-associated receptor 1 (TAAR1) by a bioluminescence resonance energy transfer cAMP biosensor. Mol Pharmacol 74(3):585-594

Barnes NL et al (2005) Absence of HER4 expression predicts recurrence of ductal carcinoma in situ of the breast. Clin Cancer Res 11(6):2163-2168

Borowsky B et al (2001) Trace amines: identification of a family of mammalian G protein-coupled receptors. Proc Natl Acad Sei USA 98(16):8966-\$9711

Brix K, Führer D, Biebermann H (2011) Molecules important for thyroid hormone synthesis and action-known facts and future perspectives. Thyroid Res 4(Suppl 1):S9

Bunzow JR et al (2001) Amphetamine, 3,4-methylenedioxymethamphetamine, lysergic acid diethylamide, and metabolites of the catecholamine neurotransmitters are agonists of a rat trace amine receptor. Mol Pharmacol 60(6): 11181-1188

Chi HC et al (2013) Thyroid hormone receptor inhibits hepatoma cell migration through transcriptional activation of Dickkopf 4. Biochem Biophys Res Commun 439(1):60-65

Ditsch N et al (2010) Thyroid function in breast cancer patients. Anticancer Res 30(5): 11713-1717

Ditsch N et al (2013) Thyroid hormone receptor (TR)alpha and TRbeta expression in breast cancer. Histol Histopathol 28(2):227-237

Elston EW, Ellis IO (1993) Method for grading breast cancer. J Clin Pathol 46(2): 189-190

Ferlay $\mathrm{J}$ et al (2015) Cancer incidence and mortality worldwide: sources, methods and major patterns in GLOBOCAN 2012. Int J Cancer 136(5)):E359-E386

Gozal EA et al (2014) Anatomical and functional evidence for trace amines as unique modulators of locomotor function in the mammalian spinal cord. Front Neural Circuits 8:134

Harbeck N, Gnant M (2017) Breast cancer. Lancet 389(10074): 1134-1150

Harmeier A et al (2015) Trace amine-associated receptor $\mathbb{1}$ activation silences GSK3beta signaling of TANAR1 and D2R heteromers. Eur Neuropsychopharmacol 25(11):2049-2061

Heublein $S$ et al (2015) Thyroid hormone receptors predict prognosis in BRCA1 associated breast cancer in opposing ways. PLoS One 10(6):e0127072

Karamouzis MV, Badra FA, Papavassiliou AG (2007) Breast cancer: the upgraded role of HER-3 and HER-4. Int J Biochem Cell Biol 39(5):851-856

Kuijpens JL et al (2005) Hypothyroidism might be related to breast cancer in post-menopausal women. Thyroid 15(11): 1253-1259

Lam VM et al (2015) In-vivo pharmacology of trace-amine associated receptor 1 . Eur J Pharmacol 763(Pt B):136-142

Le Van Thai A et al (1993) Identification of a neuron-specific promoter of human aromatic L-ammino acid decarboxylase gene. Brain Res Mol Brain Res 177(3-4):227-238

Mates $M$ et al (2015) Systemic targeted therapy for her2-positive early female breast cancer: a systematic review of the evidence for the 2014 Cancer Care Ontario systemic therapy guideline. Curr Oncol 22(Suppl 1):S1114-S122

Mitri Z, Constantine T, O'Regan R (2012) The HER2 receptor in breast cancer: pathophysiology, clinical use, and new advances in therapy. Chemother Res Pract 2012:743193

Naresh A et al (2006) The ERBB4/HER4 intracellular domain 4ICD is a BH3-only protein promoting apoptosis of breast cancer cells. Cancer Res 66(12):6412-6420

Pan B, Huang XF, Deng C (2011) Antipsychotic treatment and neuregulin 1-ErbB4 signalling in schizophrenia. Prog Neuropsychopharmacol Biol Psychiatry 35(4):924-930
Perri A et al (2014) T3 enhances thyroid cancer cell profiferation through TRbetal//Octt-1-mediated eyefin DI activation. Mol Cell Endocrinol 382(1):205-2117

Pestka A et al (2013) Nuclear hormone receptors and female reproduction. Curr Mol Med 13(7):1066-1078

Pons R et al (2004) Aromatic L-ammino acid decarboxylase deficiency: clinical features, treatment, and prognosis. Neurology 62(7): 1058-1065

Raab $S$ et al (2016) Incretin-fike effects of small molecule trace amine-associated receptor $\mathbb{1}$ agonists. Mol Metab 5(1):47-56

Rasmusson B et al (1987) Thyroid function in patients with breast cancer. Eur J Cancer Clin Oncol 23(5):553-556

Remmele W, Stegner HE (1987) Recommendation for uniform definition of an immunoreactive score (IRS) for immunohistochemical estrogen receptor detection (ER-ICA) in breast cancer tissue. Pathologe 8(3): 138-140

Scanlan TS et al (2004) 3-Iodothyronamine is an endogenous and rapid-acting derivative of thyroid hormone. Nat Med 10(6)::638-642

Sogaard M et al (2016) Hypothyroidism and hyperthyroidism and breast cancer risk: a nationwide cohort study. Eur J Endocrinol 174(4):-409-414

Sotnikova TD, Caron MG, Gainetdinov RR (2009) Trace amine-associated receptors as emerging therapeutic targets. Mol Pharmacol 76(2):229-235

Sriram U et al (2016) Methamphetamine induces trace amine-associated receptor $\mathbb{1}(T \mathbb{A} \mathbb{R}$ ) expression in human $T$ lymphocytes: role in immunomodulation. J Leukoc Biol 99(1):213-223

Sumi-Ichinose C et al (1992) Molecular cloning of genomic DNA and chromosomal assignment of the gene for human aromatic L-ammino acid decarboxylase, the enzyme for catecholamine and serotonin biosynthesis. Biochemistry 31(8):2229-2238

Tan M, Yu D (2007) Molecular mechanisms of erbB2-mediated breast cancer chemoresistance. Adv Exp Med Biol 608:119-129

The Human Metaholome Database (2016) Aromatic-L-amino-acid decarboxylase. Available from: http://www.hmdb.ca/proteins/ HMDBP00278. Accessed 20 Nov 2016

Torre LA et al (2015) Global cancer statistics, 2012. CA Cancer J Clin 65(2):87-108

Turken O et al (2003) Breast cancer in association with thyroid disorders. Breast Cancer Res 5(5):R1110-R113

USFaD Administration (2016) Available from: http://www.fda.gov/ Drugs/InformationOnDrugs/ApprovedDrugs/ucm370449.htm. Accessed 20 Nov 2016

Vidal GA et al (2005) Presenilin-dependent gamma-secretase processing regulates multiple ERBB4/HER4 activities. J Biol Chem 280(20): 197777-19783

Wang C, Liu Y, Cao JM (2014) G protein-coupled receptors: extranuclear mediators for the non-genomic actions of steroids. Int $\mathbf{J}$ Mol Sci 15(9):154112-15425

Witzel I, Muller V (2015) Targeted therapies in breast cancer: new approaches and old challenges. Breast Care (Basel) 10(3): 1157-158

Yarden Y, Sliwkowski MX (2001) Untangling the ErbB signalling network. Nat Rev Mol Cell Biol 2(2): 127-137

Yokoe $T$ et al (1996) Relationship between thyroid-pituitary function and response to therapy in patients with recurrent breast cancer. Anticancer Res 16(4A):2069-2072

Zoi I et al (2016) RANKL signaling and ErbB receptors in breast carcinogenesis. Trends Mol Med 22(10):839-850 